\title{
Bajos niveles de actividad de la lipasa ácida lisosomal y su relación con el desarrollo de la cirrosis de origen criptogénica/NASH: un estudio de cohorte
}

\author{
Luis Guillermo Toro Rendon, Luisa Fernanda Calle Tavera, Elizabeth María Correa Gutiérrez, \\ Adriana Ocampo Mesa, Jaime Alberto Ramírez Arbeláez \\ Hospitales de San Vicente Fundación. Medellín - Rionegro, Colombia.
}

Acta Gastroenterol Latinoam 2021;51(1):57-63

Recibido: 16/11/2020 / Aceptado: 14/02/2021 / Publicado online: 22/03/2021 / https://doi.org/10.52787/zhju9301

\section{Resumen}

Introducción y objetivo. La deficiencia de lipasa ácida lisosomal (LAL-D) se puede relacionar con cirrosis por esteatohepatitis no alcohólica (NASH). Muchas de las cirrosis clasificadas como criptogénicas son la evolución de una enfermedad hepática por hígado graso. El objetivo del presente estudio fue evaluar la actividad de la lipasa ácida lisosomal (LAL) en los pacientes con cirrosis hepática de cualquier etiología y establecer si los niveles bajos se correlacionan con la cirrosis de origen criptogénica/NASH. Métodos. Fue un estudio de cohorte analítico. Se incluyeron 96 pacientes con cirrosis de cualquier etiología a los cuales se les midió la actividad de la LAL. Resultados. Se incluyeron a 55 pacientes con cirrosis de origen criptogénica/NASH y 41 de otras etiologías. El 53\% de la población total era mujer. El 58\% tenía un diagnóstico de cirrosis criptogénical NASH. Los scores de severidad de la enfermedad hepática fueron: MELD (11,72 \pm 7,3 vs. 7,14 $\pm 5,7 ; p=0,001)$ $y$ Child-Pugh $(7,26 \pm 3,8$ vs. $4,34 \pm 3,8 ; p=0,004)$ fueron significativamente más altos en los pacientes con la cirrosis criptogénicalNASH. La actividad de la LAL fue significativa menor $(202,40 \pm 98,8$ vs. $242,55 \pm 121$,9; $p=0,04)$ en el grupo de cirrosis criptogénica/NASH. En el análisis multivariable, la baja actividad de la LAL (< $150 \mathrm{nmol} / \mathrm{spot} / \mathrm{hora}$ ) se correlacionó con la presencia de la cirrosis criptogénica/NASH. Conclusiones. los pacientes con cirrosis de origen criptogénica/NASH tienen niveles de actividad de LAL más reducidos que aquellos con cirrosis de otras etiologías. La actividad de la LAL por debajo de $150 \mathrm{nmol} / \mathrm{spot} / \mathrm{hora}$ es un factor de riesgo para el desarrollo de la cirrosis de origen criptogénica/NASH.

Palabras claves. Lipasa ácida lisosomal, cirrosis, cirrosis criptogénica, esteatohepatitis no alcohólica, enfermedad de Wolman.

\section{Low Levels of Lysosomal Acid Lipase Activity and Its Relationship with the Development of Cirrhosis of the Cryptogenic Origin or NASH: a Cohort Study}

\section{Summary}

Introduction and objective. The deficiency of the Lysosomal acid lipase (LAL) activity has been related to cirrhosis due to non-alcoholic steatohepatitis (NASH). Many of the cirrhosis classified as cryptogenic are the evolution of liver disease due to fatty liver. The objective of the present study
Correspondencia: Luis Guillermo Toro Rendon

Correo electrónico: lgtoro2101@gmail.com 
was to evaluate the lysosomal acid lipase (LAL) activity in the patients with liver cirrhosis of any etiology and establish whether low levels correlate with cryptogenic cirrhosis of origin cryptogenic or NASH. Methods. Was an analytical cohort study including 96 patients with cirrhosis of any etiology for which LAL activity was measured. Results. Fifty-five patients (58\%) with cryptogenic cirrhosis or NASH were included and 41 with other etiologies. The fifty-three percent of the total population were women. The severity scores of liver disease were significantly higher in the patients with the cryptogenic cirrhosis or NASH: MELD (11.72 \pm 7.3 vs $4,34 \pm 5.7 ; p=0.001)$ and Child-Pugh (7.26 \pm 3.8 vs $7.26 \pm 3.8 ; p=0.004)$. LAL activity was significant lower $(202.40 \pm 98.8$ vs $242.55 \pm 121.9 ; p=0.04)$ in the cirrhosis cryptogenic or NASH group. In the multivariate analysis, low LAL activity (< 150 nmollspot/hour), was correlated with the presence of cryptogenic cirrhosis or NASH. Conclusions. The patients with cryptogenic cirrhosis or NASH have lower levels of LAL activity than those with cirrhosis of other etiologies. $L A L$ activity below $150 \mathrm{nmol} / \mathrm{spot} / \mathrm{hour}$ is predictive of the cryptogenic cirrhosis or NASH.

Keywords. Lysosomal acid lipase, cirrhosis, cryptogenic cirrhosis, non-alcoholic steatohepatitis, Wolman's disease.

\section{Abreviaturas}

LAL-D: Deficiencia de lipasa ácida lisosomal.

LAL: Lipasa ácida lisosomal.

VLDL: Lipoproteina de muy baja densidad.

LDL: Lipoproteinas de baja densidad.

HDL: Lipoproteinas de alta densidad.

EAEC: Enfermedad por almacenamiento de ésteres de colesterol.

NASH: Esteatohepatitis no alcohólica.

Anti-VHC: Anticuerpos contra el virus de la hepatitis $C$.

HBsAg: Antígeno de superficie de hepatitis $B$.

ANA: Anticuerpos antinucleares.

AMA: Anticuerpos mitocondriales.

ASMA: Anticuerpos contra el músculo liso.

AIC: Criterio de información Akaike.

HTA: Hipertensión arterial.

DM: Diabetes mellitus.

IMC: Indice de masa corporal.

MELD: Model for End-Stage Liver Disease.

\section{Puntos claves. Mensajes de aprendizaje}

- La LAL-D profunda se asocia con el desarrollo de la cirrosis temprana.

- Una gran proporción de las cirrosis catalogadas como criptogénicas son en realidad cirrosis por NASH.
- En este estudio se encontró una correlación entre los niveles bajos de actividad de la LAL y la cirrosis criptogénica/NASH.

- La LAL-D puede ser un marcador de la enfermedad hepática más severa en los pacientes con cirrosis por NASH.

- Ante los resultados encontrados en este estudio, se plantea la posibilidad de que, así como sucede con la LAL-D, los bajos niveles de LAL también podrían ocasionar una acumulación de colesterol y triglicéridos a nivel de los lisosomas hepáticos, disminuyendo la producción de colesterol libre y de ácidos grasos, favoreciendo el desarrollo de la NASH y la posterior presentación de la cirrosis.

\section{Introducción}

La deficiencia de lipasa ácida lisosomal (LAL-D) es una rara enfermedad autosómica recesiva de depósito de lípidos, ${ }^{1}$ causada por la mutación del gen LIPA, el cual está localizado en el cromosoma 10q23.2. Produce una eliminación o una reducción marcada de la actividad de la enzima lipasa ácida lisosomal (LAL). ${ }^{2}$ Esta enfermedad puede encontrarse no solo en la infancia, sino también en la edad adulta avanzada, puesto que depende de la actividad enzimática residual. ${ }^{3}$

La LAL-D lleva a una ausencia o disminución en la hidrólisis de los ésteres de colesterol y triglicéridos, con un aumento en la producción de la apolipoproteína B y lipoproteína de muy baja densidad (VLDL), lo que explica porque la dislipidemia observada en individuos con la LAL-D se caracteriza por colesterol total y LDL elevado, así como por las lipoproteínas de alta densidad (HDL) muy bajas. ${ }^{4}$

Esta falla en la degradación ocasiona un almacenamiento a nivel sistémico de los ésteres de colesterol y los triglicéridos en los lisosomas de varios tejidos y tipos celulares, predominando en el hígado, bazo, tracto gastrointestinal y paredes de los vasos sanguíneos. ${ }^{5}$ Esta acumulación es un potente inductor de fibrosis, lo que lleva a un incremento en la frecuencia y al desarrollo temprano de la cirrosis en estos pacientes. ${ }^{2,6}$

Aunque hay más de 40 mutaciones del gen LIPA en la enfermedad de Wolman y en la enfermedad por almacenamiento de ésteres de colesterol (EAEC), la mutación que más comúnmente ha sido reportada es la c. $894 \mathrm{G}>\mathrm{A}$ : E8SJM. La verdadera prevalencia es desconocida, varía según la raza, pero se ha estimado que puede estar entre 1:528.000 en los recién nacidos y de 1:130.000 a 1:370.000 en los niños y adultos. ${ }^{7} 8$

En los lactantes, se presenta la manifestación más severa de la enfermedad. Conocida como enfermedad de 
Wolman, la actividad de la LAL es $<1 \%$ o está completamente ausente y su progresión es rápida. Tiene una media de edad para la primera presentación de 1 mes y, posteriormente, la muerte ocurre por lo regular alrededor de los 6 meses de vida por una falla multiorgánica y el compromiso de la función hepática secundario a la cirrosis. ${ }^{9}$

Las primeras descripciones de la EAEC se atribuyen a Fredrickson, ${ }^{10}$ Schiff,,${ }^{11}$ Lageron ${ }^{12}$ e Infante. ${ }^{13}$ En estos, la progresión de la enfermedad es más lenta, hay una actividad residual de la LAL (1-12\% de la actividad normal), lo que conduce a una cirrosis y otras complicaciones en la infancia o en etapas más avanzadas de la vida. ${ }^{6}{ }^{14}$ La primera manifestación clínica, por lo regular, se encuentra en la infancia, pero el diagnóstico puede retrasarse hasta etapas avanzadas de la enfermedad (por la actividad residual de la LAL). Suelen presentar un compromiso hepático, el cual puede progresar a una cirrosis y, luego, requerir un trasplante hepático. ${ }^{15}$

La información de la cual se dispone sobre las manifestaciones clínicas de la EAEC se basa en los reportes de casos, las pequeñas series de casos o la revisión de la literatura. ${ }^{16}$ En ocasiones, esta entidad puede pasar inadvertida hasta la edad adulta, momento en el cual se puede presentar con un amplio espectro de enfermedad hepática que puede ir desde la hepatomegalia con hígado graso (en más del $90 \%$ de los casos), hasta un desarrollo silente de fibrosis que evoluciona a la cirrosis. ${ }^{9,17}$ La esplenomegalia es más variable (74\% de los pacientes), muy posiblemente secundaria a la hipertensión portal. ${ }^{9,} 18$ También es posible encontrar manifestaciones de aterosclerosis en diferentes territorios vasculares y la enfermedad cardiovascular prematura. ${ }^{9,17}$

La sospecha de la LAL-D se basa en los antecedentes médicos, los hallazgos clínicos y los resultados de las pruebas bioquímicas. El diagnóstico se realiza mediante la demostración del déficit enzimático, la cual se puede realizar en diferentes tipos de muestras (leucocitos en sangre periférica, fibroblastos o gota de sangre seca). ${ }^{?}$

La cirrosis criptogénica se define como aquella en la cual, luego de un estudio clínico y paraclínico detallado, no se encuentra una etiología exacta. ${ }^{19}$ Da cuenta del 5-30\% de todas las causas de cirrosis y de aproximadamente el 10\% de los trasplantes hepáticos. ${ }^{20}$ Se considera que la cirrosis criptogénica, en muchos casos, podría ser la evolución de la esteatohepatitis no alcohólica (NASH). De hecho, el 25\% de los pacientes afectados por NASH puede desarrollar cirrosis. ${ }^{21,22}$

Se ha sugerido que la reducción en la actividad de la LAL también podría ser un factor contribuyente no reconocido en el desarrollo y progresión de la enfermedad hepática grasa no alcohólica a la cirrosis criptogénica. ${ }^{23}$

El objetivo de este estudio fue determinar si los niveles bajos en la actividad de la LAL se correlacionan con la presencia de cirrosis de origen criptogénica/NASH en comparación con una cirrosis de cualquier otra etiología.

\section{Materiales y métodos}

\section{Diseño y escenario del estudio}

Este es un estudio observacional de cohorte, prospectivo y analítico, realizado en un hospital de cuarto nivel de atención, desde julio de 2017 a enero de 2018. Se tomó una muestra por conveniencia en la cual se reclutaron a 96 pacientes mayores de 14 años con el diagnóstico de cirrosis (imagenológico o por biopsia) de cualquier etiología que asistieron de manera consecutiva y fueron valorados en el servicio de hepatología de los Hospitales de San Vicente Fundación (Medellín - Rionegro). Ellos aceptaron ingresar en el estudio y firmaron el consentimiento informado antes de la toma de muestra para el análisis de la actividad enzimática. Se excluyeron aquellos pacientes que tenían evidencia de la enfermedad metastásica hepática.

Para el análisis se dividió la población en dos grupos. El primero corresponde a los pacientes con cirrosis por NASH/criptogénica. La cirrosis por NASH se definió por la presencia de historia previa de enfermedad por hígado graso, sin presencia de otras etiologías posibles o por los hallazgos en la biopsia hepática. La cirrosis criptogénica fue definida como la ausencia de antecedentes de consumo de alcohol (> $20 \mathrm{gr} / \mathrm{dí}$ en mujeres y > $30 \mathrm{gr} /$ día en hombres), anticuerpos contra el virus de la hepatitis C (Anti-VHC) y antígeno de superficie de hepatitis B (HBsAg) negativos; anticuerpos antinucleares (ANA) < 1:80; anticuerpos mitocondriales (AMA): negativos; anticuerpos contra el músculo liso (ASMA): negativos; saturación de transferrina, niveles séricos de ceruloplasmina y alfa-1 antitripsina: normales. En el segundo grupo se incluyeron los pacientes con una cirrosis de cualquier otra etiología con diagnóstico ya establecido (imagen o biopsia).

El protocolo del estudio estaba planeado para los periodos de julio de 2017 - julio 2018, pero se debió suspender de manera prematura, luego de 6 meses de su inicio por un cambio en el laboratorio de referencia y en la técnica de medición de la actividad de la LAL.

En la primera consulta, a todos los pacientes se les realizó una evaluación clínica, exploración física y se consignaron los datos sobre las características demográficas.

\section{Toma de muestra y análisis de la actividad enzimática de la LAL}

En la visita de ingreso al estudio se obtuvo una muestra de gota de sangre venosa para la medición de la acti- 
vidad de la LAL, la cual fue tomada por una punción del pulpejo, se dispuso en un papel de filtro y se guardó a temperatura ambiente para enviar al laboratorio de referencia (The Children's Hospital of Seattle) donde se midió la actividad enzimática.

\section{Análisis estadístico}

El análisis estadístico se realizó con el uso del software R versión 3.3.2 y el paquete Office de Microsoft. El análisis descriptivo se realizó utilizando frecuencias relativas. Las variables continuas se compararon con el uso de pruebas $\mathrm{T}$ de Student no emparejadas y se informaron como medias con desviaciones estándar; las variables categóricas se compararon con el uso de pruebas de la chi-cuadrado y se informaron como frecuencias y porcentajes. Se consideró que los valores de $p$ inferiores a 0,05 indicaban significación estadística.

El análisis multivariable se ajustó con un modelo de regresión logística cuya variable respuesta fue la presencia (sí-no) de la cirrosis criptogénica/NASH. Inicialmente, se utilizaron los niveles de LAL como principal variable explicativa; de forma posterior, introdujeron el siguiente grupo de variables para evaluar su relación con la variable respuesta y, así mismo, su evaluación como posibles confusoras y de interacción. El mejor modelo se eligió utilizando el criterio de información Akaike o AIC por sus siglas en inglés (Akaike information criterion). El ajuste del modelo logístico se realizó con la prueba de Hosmer Lemeshow. La interacción fue evaluada realizando una ANOVA comparando el modelo sin y con la variable de interacción.

\section{Resultados}

Entre julio de 2017 y enero de 2018, se incluyeron en el estudio a 96 pacientes. El 58\% $(\mathrm{n}=55)$ tenía cirrosis criptogénica/NASH y en el $42 \%(\mathrm{n}=44)$ la cirrosis era de otra etiología.

En la población total, el 53\% $(\mathrm{n}=53)$ era mujer, con una edad promedio para el sexo femenino de 58,62 años y para el masculino de 60,35 años. Las características demográficas de la población se muestran en la Tabla 1 .

En los pacientes que tenían definida la etiología de

Tabla 1. Características demográficas y clínicas de los pacientes de acuerdo a la etiología de la cirrosis

\begin{tabular}{|c|c|c|c|}
\hline Variables & $\begin{array}{c}\text { Cirrosis } \\
\text { criptogénica/NASH } \\
(n=55)\end{array}$ & $\begin{array}{l}\text { Otro tipo de } \\
\text { cirrosis } \\
(\mathrm{n}=41)\end{array}$ & $P$ valor** \\
\hline \multicolumn{4}{|l|}{ Características clínicas } \\
\hline Edad (años) ${ }^{*}$ & $59,53 \pm 11,4$ & $54,48 \pm 15,5$ & 0,07 \\
\hline Mujer (\%) & 66,66 & 46,93 & 0,05 \\
\hline Hipertensión arterial (\%) & 55,55 & 30,61 & 0,02 \\
\hline Diabetes mellitus (\%) & 53,33 & 24,48 & 0,007 \\
\hline Índice de masa corporal* & $29,57 \pm 5,9$ & $26,94 \pm 5,13$ & 0,02 \\
\hline \multicolumn{4}{|l|}{ Laboratorios* } \\
\hline LAL (nmol/spothora) & $202,40 \pm 98,8$ & $242,55 \pm 121,9$ & 0,04 \\
\hline Colesterol total (mg/dL) & $156,55 \pm 36,7$ & $169,23 \pm 48,8$ & 0,07 \\
\hline Colesterol de alta densidad (HDL) (mg/dL) & $40,88 \pm 9,6$ & $43,71 \pm 12,7$ & 0,11 \\
\hline Creatinina (mg/dL) & $1,75 \pm 4,9$ & $0,93 \pm 0,3$ & 0,24 \\
\hline Triglicéridos (mg/dL) & $125,7 \pm 56,9$ & $141,49 \pm 69,6$ & 0,21 \\
\hline Gamma glutamil transpeptidasa (IU/L) & $193,56 \pm 160,6$ & $126,98 \pm 112,34$ & 0,03 \\
\hline Fosfatasa alcalina (UI/L) & $198,35 \pm 137,6$ & $184,53 \pm 177$ & 0,64 \\
\hline Bilirrubina total (mg/dL) & $2,08 \pm 2,02$ & $2,05 \pm 4,32$ & 0,87 \\
\hline Bilirrubina directa (mg/dL) & $1,03 \pm 1,05$ & $1,15 \pm 1,81$ & 0,73 \\
\hline Transaminasa glutámico-pirúvica (ALT) (U/L) & $40,72 \pm 21,5$ & $49,75 \pm 30,5$ & 0,09 \\
\hline Transaminasa glutámico-oxalacética (AST) (U/L) & $56,6 \pm 30,4$ & $51,06 \pm 30,3$ & 0,68 \\
\hline Albúmina (mg/dL) & $3,46 \pm 0,66$ & $3,75 \pm 0,7$ & 0,07 \\
\hline INR & $1,29 \pm 0,24$ & $1,21 \pm 0,19$ & 0,76 \\
\hline Glucosa (mg/dL) & $121,92 \pm 52,8$ & $105,30 \pm 28,2$ & 0,03 \\
\hline
\end{tabular}

INR: Índice internacional normalizado (International normalized ratio). 
Tabla 1

\begin{tabular}{lccc}
\hline Variables & $\begin{array}{c}\text { Cirrosis } \\
\text { criptogénica/NASH } \\
(\mathbf{n}=\mathbf{5 5})\end{array}$ & $\begin{array}{c}\text { Otro tipo de } \\
\text { cirrosis } \\
(\mathbf{n}=\mathbf{4 1})\end{array}$ & $\boldsymbol{P}^{\text {valor**}}$ \\
\hline Plaquetas $(\mathrm{x} 1000 / \mathrm{ll})$ & $119,67 \pm 59,8$ & $203,88 \pm 120,7$ & $<0,001$ \\
Hemoglobina $(\mathrm{g} / \mathrm{dL})$ & $13,12 \pm 2,1$ & $13,57 \pm 2,1$ & 0,31 \\
Ferritina $(\mathrm{ng} / \mathrm{mL})$ & $228,15 \pm 220,5$ & $173,64 \pm 140,7$ & 0,15 \\
Hormona estimulante de tiroides (TSH) (mUI/L) & $3,46 \pm 2,35$ & $3,91 \pm 2,97$ & 0,47 \\
Severidad de la enfermedad hepática* & & & 0,001 \\
MELD & $11,72 \pm 7,3$ & $7,14 \pm 5,7$ & 0,004 \\
Child-Pugh (puntos) & $7,26 \pm 3,8$ & $4,34 \pm 3,8$ & \\
\hline
\end{tabular}

* Media \pm DE.

${ }^{\star *}$ T de Student para muestras independientes, chi-cuadrado para diferencia de proporciones.

Significancia estadística: $p<0,05$.

la cirrosis, la causa más común fue el origen alcohólico (22\%). En la Tabla 2 se muestran las causas de cirrosis en los pacientes del estudio.

La prevalencia de la HTA $(55,5 \%$ vs. $30,61 \%$; $p=0,02)$ y la DM $(53,33 \%$ vs. $24,48 \% ; p=0,007)$ fue significativamente mayor en el grupo de pacientes con la cirrosis por NASH/criptogénica. El índice de masa corporal (IMC) $(29,57 \pm 5,9$ vs. $26,94 \pm 5,13 ; p=0,02)$ fue más elevado en aquellos pacientes con la cirrosis por NASH/criptogénica en la comparación con la de otras etiologías.

No se encontró diferencia estadísticamente significativa entre los dos grupos en la actividad necroinflamatoria hepática dada por los valores de transaminasas.

Los scores de severidad de la enfermedad hepática fueron: MELD (11,72 $\pm 7,3$ vs. 7,14 $\pm 5,7 ; p=0,001)$ y Child-Pugh $(7,26 \pm 3,8$ vs. $4,34 \pm 3,8 ; p=0,004)$ fueron significativamente más altos en los pacientes con cirrosis criptogénica/NASH. La actividad de la LAL fue menor en forma significativa $(202,40 \pm 98,8$ vs. $242,55 \pm 121,9 ; p=0,04)$ en el grupo de pacientes con cirrosis criptogénica/NASH.

Tabla 2. Etiología de la cirrosis

\begin{tabular}{lcc}
\hline Causa de la cirrosis & $\mathrm{n}$ & $\%$ \\
\hline Criptogénica/NASH & 55 & 58 \\
Hepatitis B & 5 & 5 \\
Hepatitis C & 2 & 2 \\
Autoinmune & 10 & 10 \\
Alcohol & 21 & 22 \\
Otras & 3 & 3 \\
Total & 96 & 100 \\
\hline
\end{tabular}

En el análisis multivariable, encontramos que las mujeres, los pacientes diabéticos, el IMC elevado y presentar niveles de $\mathrm{LAL}<150 \mathrm{nmol} /$ spot/hora, tienen una relación significativa con la cirrosis criptogénica/NASH (Tabla 3). $\mathrm{Al}$ evaluar la edad como variable de confusión, incluyéndola en el modelo, y al realizar la comparación basada en

Tabla 3. Modelo de regresión logística

\begin{tabular}{lcl}
\hline Variables & OR (IC 95\%) & $P$ valor \\
\hline Mujer & $3,98(1,51-11,58)$ & 0,001 \\
Diabetes & $4,32(1,63-12,34)$ & 0,004 \\
IMC & $1,1 \quad(1,01-1,22)$ & 0,02 \\
Lipasa $<150$ & $3,68(1,36-10,75)$ & 0,01 \\
\hline
\end{tabular}

residuales, incluida o no en el modelo, no se encontró suficiente evidencia para demostrar que el modelo con la edad era mejor que sin ella; así mismo, el valor del beta de la variable actividad de la LAL no tuvo una diferencia superior al $10 \%$, con lo cual, se concluyó que la edad no fue una variable confusora.

La interacción de la diabetes mellitus (DM) con la actividad de la LAL se evaluó realizando una ANOVA, comparando el modelo con y sin la variable de interacción, obteniendo un valor $p=0,09$, concluyendo que no había suficiente evidencia para demostrar que la diabetes tuviera una interacción con la actividad de la LAL.

Para evaluar el ajuste del modelo logístico, se utilizó la prueba de Hosmer Lemeshow que, con un valor de $p=0,73$, ofreció suficiente evidencia para rechazar la hipótesis nula a favor de que el modelo tuvo un buen ajuste y que fue explicado adecuadamente por los datos. 


\section{Discusión y conclusiones}

La enfermedad hepática grasa no alcohólica ocurre en todos los grupos de edad y su prevalencia se incrementa con el aumento del peso corporal. El hígado graso se encuentra en el 10\%-15\% de los individuos de peso normal y en el $70 \%$ de los sujetos obesos. ${ }^{24}$ Esta entidad está estrechamente relacionado con la obesidad, pero también se encuentra de manera frecuente en pacientes con la DM tipo 2 y dislipidemia, con una prevalencia que va del 50\%-90\% en estos subgrupos de pacientes. ${ }^{25}$ En la actualidad la NASH es una causa frecuente de cirrosis y trasplante hepático.

En hígados explantados de pacientes que fueron llevados a trasplante hepático y tenían diagnóstico previo de cirrosis de origen criptogénica, se demostró que un gran porcentaje $(25 \%)$ en realidad lo que tenía era una cirrosis por NASH. ${ }^{26}$

La LAL-D es una entidad rara que puede estar subdiagnosticada en virtud a su baja prevalencia y a que tanto las características clínicas, bioquímicas, como los hallazgos en la patología pueden ser encontrados en otras entidades mucho más comunes. La progresión de la enfermedad hepática en niños y pacientes adultos con la LAL-D es más acelerada y la evolución natural de estos pacientes muestra que tienen un alto riesgo de trasplante de hígado. ${ }^{8}$

En un estudio multicéntrico de Angelico et al., se encontró que, de 274 pacientes con diferentes causas de cirrosis, 133 (48\%) tenían cirrosis criptogénica. A los participantes del estudio, se les midió la actividad de la LAL y se encontró una reducción significativa de la actividad enzimática en el grupo de pacientes con cirrosis criptogénica, cuando se comparó con otras etiologías de cirrosis. ${ }^{22}$

De forma similar a los hallazgos encontrados por Angelico et al., Espasiani-Gentilucci et al. describieron que niveles bajos de la LAL-D se encontraban en los pacientes con cirrosis de origen criptogénica al ser comparados con personas sanas o con cirróticos de otras etiologías. ${ }^{27}$

En nuestro estudio encontramos que aquellos pacientes con cirrosis de origen criptogénica/NASH cursaban con una enfermedad más severa medida por los scores de Child-Pugh y MELD. De forma análoga a lo reportado por Angelico et al., y a lo esperado por su asociación con la enfermedad hepática grasa no alcohólica, se encontró una mayor prevalencia de la HTA y la DM, sin demostrar una relación estadísticamente significativa con el sexo o la edad.

En el grupo de pacientes con NASH/criptogénica, los niveles de actividad de la LAL fueron estadísticamente más bajos de forma significativa que en el grupo de pacientes con cirrosis de otras etiologías. El análisis multivariado demostró que en aquellos pacientes con niveles de actividad de la $\mathrm{LAL}<\mathrm{a} 150 \mathrm{nmol} /$ spot/hora, era 3,68 veces más posible que cursaran una cirrosis de origen
criptogénica/NASH que aquellos con niveles superiores. En ninguno de los pacientes del estudio se investigó la mutación E8SJM, ya que no cursaban con niveles tan bajos que justificaran realizar este análisis.

Si bien en nuestro estudio encontramos una correlación entre los niveles bajos de actividad de la LAL y la cirrosis criptogénica/NASH, los valores encontrados no fueron tan bajos como los reportados en otros estudios. ${ }^{22,27}$

Este estudio tiene algunas limitaciones. Algunos son el tamaño de la muestra y la imposibilidad para poder concluirlo según se había estipulado en el protocolo debido al cambio de proveedor de medición de la prueba, lo cual disminuyó de manera significativa el tamaño de la población que se pretendía captar.

Se requieren estudios futuros con un mayor poder que evalúen la actividad de la LAL y su asociación con la cirrosis de origen criptogénica/NASH y que demuestren si la LAL-D puede ser un marcador de enfermedad hepática más severa en este grupo de pacientes.

\section{Conclusión}

La LAL-D se puede considerar como una causa emergente de hígado graso y disfunción hepática. Las características comunes de la LAL-D y la enfermedad por hígado graso en adultos incluyen niveles elevados de aminotransferasas, dislipidemia, hepatomegalia, fibrosis hepática y cirrosis; por lo que, en la población de pacientes con NASH y cirrosis criptogénica, el diagnóstico de LAL-D puede pasar desapercibido.

Los pacientes con cirrosis de origen criptogénica/ NASH tienen niveles de actividad de LAL más reducidos que aquellos con cirrosis de otras etiologías y cursan con una enfermedad hepática más severa, medida por los scores de Child-Pugh y MELD.

La actividad de la LAL por debajo de $150 \mathrm{nmol} /$ spot/ hora es un factor de riesgo para el desarrollo de la cirrosis de origen criptogénica/NASH.

\section{Conflictos de intereses y responsabilidad intelectual.} Los autores de este estudio asumen la responsabilidad total del contenido del manuscrito y declaran que los datos, modelos y metodología utilizados en la investigación son de su propiedad intelectual. Además, declaran no tener conflictos de intereses.

Sostén financiero. El costo del procesamiento de la actividad enzimática fue asumido por Alexion Pharmaceuticals quien no tuvo ninguna injerencia sobre los datos o los resultados del estudio. 
Consideraciones éticas. El presente estudio se considera dentro de la categoría de investigación de riesgo minimo, pues antes de proceder con la inclusión de los pacientes y la recolección de datos, fue aprobado por el comité de ética de la investigación de los Hospitales de San Vicente Fundación. Se tomó consentimiento informado a todos los pacientes.

\section{Aviso de derechos de autor}

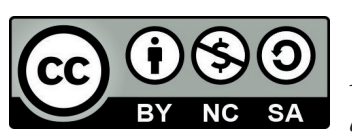

(C) 2021 Acta Gastroenterológica Latinoamericana. Este es un artículo de acceso abierto publicado bajo los términos de la Licencia Creative Commons Attribution (CC BY-NC-SA 4.0), la cual permite el uso, la distribución y la reproducción de forma no comercial, siempre que se cite al autor y la fuente original.

Cite este artículo como: Toro Rendon LG, Calle Tavera LF, Correa Gutiérrez EM y col. Bajos niveles de actividad de la lipasa ácida lisosomal y su relación con el desarrollo de la cirrosis de origen criptogénica/NASH: un estudio de cohorte. Acta Gastroenterol Latinoam. 2021;51(1):57-63. https:// doi.org/10.52787/zhju9301

\section{Referencias}

1. McKusick-Nathans Institute of Genetic Medicine, Johns Hopkins University School of Medicine. Lysosomal acid lipase deficiency. Online Mendelian Inheritance in Man [citado 13 de junio de 2020]. Disponible en: https://omim.org/entry/278000? search=lysosomal\%20acid\%20 lipase\&highlight=acid\%20lipase\%20lysosomal

2. Reiner Ž, Guardamagna O, Nair D, et al. Lysosomal acid lipase deficiency - An under-recognized cause of dyslipidaemia and liver dysfunction. Atherosclerosis. 2014;235(1):21-30.

3. Strebinger G, Müller E, Feldman A, Aigner E. Lysosomal acid lipase deficiency - early diagnosis is the key. Hepat Med. 2019;11:79-88.

4. Wilson DP, Patni N. Lysosomal Acid Lipase Deficiency. En: Feingold KR, Anawalt B, Boyce A, et al., editores. Endotext. South Dartmouth (MA): MDText.com, Inc. 2000 [citado 13 de junio de 2020]. Disponible en: http://www.ncbi.nlm.nih.gov/ books/NBK395569/

5. Bernstein DL, Hülkova H, Bialer MG, Desnick RJ. Cholesteryl ester storage disease: review of the findings in 135 reported patients with an underdiagnosed disease. J Hepatol. 2013;58(6):1230-43.

6. Pant M, Oshima K. Cholesteryl Ester Storage Disease: An underdiagnosed cause of cirrhosis in adults. Ann Diagn Pathol. 2017;31:66-70.

7. Scott SA, Liu B, Nazarenko I, et al. Frequency of the Cholesteryl Ester Storage Disease Common LIPA E8SJM Mutation (c.894G>A) in Various Racial and Ethnic Groups. Hepatology. 2013;58(3):958-65.

8. Burton BK, Silliman N, Marulkar S. Progression of liver disease in children and adults with lysosomal acid lipase deficiency. Curr Med Res Opin. 2017;33(7):1211-4.
9. Camarena C, Aldamiz-Echevarria LJ, Polo B, et al. Update on lysosomal acid lipase deficiency: Diagnosis, treatment and patient management. Med Clin (Barc). 2017;148(9):429.e1-429.e10.

10. Fredrickson DS, Sloan HR, Ferrans VJ, Demosky SJ. Cholesteryl ester storage disease: a most unusual manifestation of deficiency of two lysosomal enzyme activities. Trans Assoc Am Physicians. 1972;85:109-19.

11. Schiff L, Schubert WK, McAdams AJ, et al. Hepatic cholesterol ester storage disease, a familial disorder. I. Clinical aspects. Am J Med. 1968;44(4):538-46.

12. Lageron A, Caroli J, Stralin H, Barbier P. Cholesterolic polycoria in adults. I. Clinical and histochemical study. Presse Med. 1967;75(54):2785.

13. Infante R, Polonovski J, Caroli J. Cholesterolic polycoria in adults. II. Biochemical study. Presse Med. 1967;75(55):2829-32.

14. Burton BK, Balwani M, Feillet F, et al. A Phase 3 Trial of Sebelipase Alfa in Lysosomal Acid Lipase Deficiency. N Engl J Med. 2015;373(11):1010-20.

15. Valayannopoulos V, Mengel E, Brassier A, Grabowski G. Lysosomal acid lipase deficiency: Expanding differential diagnosis. Mol Genet Metab. 2017;120(1-2):62-6.

16. Burton BK, Deegan PB, Enns GM, et al. Clinical Features of Lysosomal Acid Lipase Deficiency. J Pediatr Gastroenterol Nutr. 2015;61(6):619-25.

17. Jeon TI, Osborne TF. SREBPs: metabolic integrators in physiology and metabolism. Trends Endocrinol Metab. 2012; 23(2): 65-72.

18. Zhang B, Porto AF. Cholesteryl ester storage disease: protean presentations of lysosomal acid lipase deficiency. J Pediatr Gastroenterol Nutr. 2013;56(6):682-5.

19. Nalbantoglu I, Jain D. Cryptogenic cirrhosis: Old and new perspectives in the era of molecular and genomic medicine. Semin Diagn Pathol. 2019;36(6):389-94.

20. Patel N, Sharma B, Samant H. Cryptogenic Cirrhosis. En: StatPearls. Treasure Island (FL): StatPearls Publishing; 2020.

21. Pais R, Barritt AS, Calmus Y, et al. NAFLD and liver transplantation: Current burden and expected challenges. J Hepatol. 2016;65(6):1245-57.

22. Angelico F, Corradini SG, Pastori D, et al. Severe reduction of blood lysosomal acid lipase activity in cryptogenic cirrhosis: A nationwide multicentre cohort study. Atherosclerosis. 2017;262:179-84.

23. Baratta F, Pastori D, Polimeni L, et al. Does Lysosomial Acid Lipase Reduction Play a Role in Adult Non-Alcoholic Fatty Liver Disease? Int J Mol Sci. 2015;16(12):28014-21.

24. Vernon G, Baranova A, Younossi ZM. Systematic review: the epidemiology and natural history of non-alcoholic fatty liver disease and non-alcoholic steatohepatitis in adults. Aliment Pharmacol Ther. 2011;34(3):274-85.

25. Fotbolcu H, Zorlu E. Nonalcoholic fatty liver disease as a multisystemic disease. World J Gastroenterol. 2016;22(16):4079-90.

26. Tardu A, Karagul S, Yagci MA, et al. Histopathological Examination of Explanted Liver After Transplantation in Patients With Cryptogenic Cirrhosis. Transplant Proc. 2015;47(5):1450-2.

27. Vespasiani-Gentilucci U, Gallo P, Piemonte F, et al. Lysosomal Acid Lipase Activity Is Reduced Both in Cryptogenic Cirrhosis and in Cirrhosis of Known Etiology. PLoS ONE. 2016;11(5):e0156113. 